\title{
Correlation and response in the Backgammon model: the Ehrenfest legacy
}

\author{
by C. Godrèche ${ }^{a, \text {, }}$ and J.M. Luck ${ }^{b}$. 2 \\ ${ }^{a}$ Service de Physique de l'État Condensé, CEA Saclay, 91191 Gif-sur-Yvette cedex, France \\ ${ }^{b}$ Service de Physique Théorique, CEA Saclay, 91191 Gif-sur-Yvette cedex, France
}

\begin{abstract}
We pursue our investigation of the non-equilibrium dynamics of the Backgammon model, a dynamical urn model which exhibits aging and glassy behavior at low temperature. We present an analytical study of the scaling behavior of the local correlation and response functions of the density fluctuations of the model, and of the associated fluctuation-dissipation ratios, throughout the $\alpha$-regime of low temperature and long times. This analysis includes the aging regime, the convergence to equilibrium, and the crossover behavior between them.
\end{abstract}

P.A.C.S. $02.50 . E y, 05.40 .+\mathrm{j}, 61.43 . \mathrm{Fs}, 75.50 . \mathrm{Lk}$

\footnotetext{
${ }^{1}$ godreche@spec.saclay.cea.fr

${ }^{2}$ luck@spht.saclay.cea.fr
} 


\section{Introduction}

There is a long tradition in Statistical Physics to illustrate conceptual problems on extremely simplified models [1]. The Ehrenfest dynamical urn model [2], which was considered by Kac as 'probably one of the most instructive models in the whole of Physics' [3], is an example of these. It was devised to elucidate the problems raised by the objections of Loschmidt and Zermelo against the $H$-theorem, and in particular to understand the fine details of how a system relaxes towards equilibrium.

The Ehrenfest model is defined as follows. Consider $N$ balls, labeled from 1 to $N$, distributed between two urns. The numbers of balls in the urns are denoted by $N_{1}$ and $N_{2}=N-N_{1}$. At each time step a ball is chosen at random (i.e., an integer between 1 and $N$ is chosen at random), and moved from the box where it is to the other box. If there was initially an unequal number of balls in each urn, the system will relax to the equilibrium state, characterized by the binomial law

$$
P\left(N_{1}\right)=\frac{1}{2^{N}}\left(\begin{array}{c}
N \\
N_{1}
\end{array}\right)
$$

such that each urn contains, on average, an equal number of balls. This model, subsequently studied by Kohlrausch and Schrödinger [4], was finally fully solved by Kac, Siegert and Hess [5, 6, 7].

The present paper belongs to the same tradition. It is devoted to the analytical study of a very simple urn model, which is actually a modern avatar of the Ehrenfest model, the so-called Backgammon model [8]. This model pertains to the recent stream of research where the study of the slow dynamics of complex glassy systems is replaced by that of simple models, most of them of mean-field type [9]. Its aim was to demonstrate that a microscopic model characterized by the absence of energy barriers was nevertheless able to exhibit a number of the characteristic features of glassy dynamics.

The Backgammon model generalizes the Ehrenfest model in two directions. First, it is easy to realize that the dynamics of the Ehrenfest model is effectively at infinite temperature, because there is no constraint on the move of the drawn ball. Therefore, in order to consider the dynamics of the model at finite temperature, one has to define an energy and impose e.g. a Metropolis rule for the move of the drawn ball: the move is allowed with probability one if the energy decreases or stays unchanged, and with probability $\exp (-\beta \Delta E)$ if it increases by $\Delta E$. The second generalization consists in taking $M$ boxes instead of two, and in going to the thermodynamical limit: $N \rightarrow \infty$, $M \rightarrow \infty$, with a fixed density $\rho=N / M$ of balls per box. These two generalizations define a class of 'thermodynamical urn models', of which the Backgammon model is a 
special case. In the Backgammon model the energy is chosen equal to minus the number of empty boxes [B].

Variants of this model have been considered, where either the choice of energy is different, or the a priori statistics of the elementary moves is defined otherwise [10, 11, 12, 13], or yet other rules are imposed [14]. Mentions of the similarity of the Backgammon model to the Ehrenfest model can also be found in refs. [15, 16].

The expected behavior of the model is easy to grasp. Denoting by $N_{d}$ the occupation number of the departure box, determined by the selected ball, and by $N_{a}$ the occupation number of the arrival box, drawn at random, four elementary moves are possible:

$$
\begin{aligned}
& \left.\begin{array}{l}
N_{d}>1, N_{a}>0: \Delta E=0 \\
N_{d}=1, N_{a}=0: \Delta E=0
\end{array}\right\} \text { (diffusion) } \\
& N_{d}=1, N_{a}>0: \Delta E=-1 \quad \text { (condensation), } \\
& N_{d}>1, N_{a}=0: \Delta E=+1 \quad \text { (evaporation). }
\end{aligned}
$$

The first two moves, corresponding to diffusion of the balls, are independent of temperature. The last two ones are respectively favored at low temperature (condensation), and at high temperature (evaporation).'

Therefore, at infinite temperature, since once a ball is drawn it is replaced in a box chosen at random, equilibrium is attained rapidly, characterized by a multinomial distribution of the balls amongst the boxes, a simple generalization of the result for the original Ehrenfest model. When temperature decreases, dynamics slows down because the rate of rejection of moves of the drawn ball increases. At equilibrium and at low temperature, a few boxes contain a large number of balls. Finally at zero temperature the only moves allowed are those for which energy decreases (condensation) or stays unchanged (diffusion). The dynamics becomes extremely slow as time passes. Indeed the ultimate stage when all boxes but two are empty meets the original Ehrenfest model with two boxes, except that now, if one box becomes empty, the dynamics stops. Such an event occurs with a probability of order $P(N)=2^{-N}$ [see eq. (1.1)], so that the time needed to empty one of the two boxes in this ultimate stage is exponentially large in $N$, and infinite in the thermodynamical limit. It is therefore clear that, at low temperature, after a transient regime, two time scales are involved in the process: a fast one, corresponding to the equilibration amongst the non-empty boxes, and a slow one, corresponding to the occurrence of the rare event of emptying a new box. This picture, though simple, accounts for the gist of the model.

\footnotetext{
${ }^{1}$ We use on purpose the same words -diffusion, condensation and evaporation- as used in the description of the elementary moves in the Kawasaki dynamics of an Ising chain [17. There is indeed a strong analogy between the latter model and a one-dimensional version of the Backgammon model.
} 
A virtue of the Backgammon model is that it allows to test a number of concepts which appear in the study of the non-equilibrium dynamics of real systems, though it is an extreme simplification of the latter. For instance, slow relaxational dynamics, aging properties, exploration of the two-time plane, violation of the fluctuation-dissipation theorem [18, etc. can be investigated in this model, either by numerical means [8], in a straightforward way, or by analytical methods, which turn out to be much harder [19, 10, 20, 21, 22, 23.

The relaxation of energy for the Backgammon model was the subject of previous studies [19, 10, 20, 21, 23]. More recently the relaxation of density has been addressed by Franz and Ritort [22], in order to investigate possible differences between the behavior of this observable and that of the energy. In the present work we give a thorough analytical study of this question. The methods used are similar to those introduced in ref. [23. Our presentation will therefore follow the lines of this reference, yet stay self-contained.

The outline of this paper is as follows. Sec. 2 defines the quantities studied in this work and presents the dynamical equations they obey. In Sec. 3 a formal solution of these equations is obtained by the method of characteristics. Sec. 4 makes contact with the original Ehrenfest model by considering the simple situation of infinite temperature. The two main sections of this work are Sec. 5, devoted to equilibrium properties, and especially Sec. 6, which contains a detailed investigation of the non-equilibrium $\alpha$-regime at low temperature. Sec. 7 gives a brief summary and a discussion.

\section{Physical quantities and their dynamical evolution}

Consider a finite system, made of $M$ boxes containing $N$ particles. Let $N_{i}(t)$ be the occupation number of box number $i$ at time $t$, i.e., the number of particles contained in that box. We have

$$
\sum_{i=1}^{M} N_{i}(t)=N .
$$

The Hamiltonian $\mathcal{H}$ and action $S$ of the system at inverse temperature $\beta$ read

$$
S \equiv \beta \mathcal{H}=-\beta \sum_{i=1}^{M} \delta_{N_{i}, 0} .
$$

Instead of addressing the more ambitious goal of describing the dynamics of the model by the configuration $\mathcal{C}=\left\{N_{1}, \ldots, N_{M}\right\}$ involving all the occupation numbers, we restrict our study to quantities involving the occupation number of one box only. We consider the thermodynamical limit $(N \rightarrow \infty, M \rightarrow \infty)$, with a fixed density $\rho=N / M$, and

furthermore restrict the analysis to the homogeneous non-equilibrium initial condition 
where there is one particle per box. We thus have $N=M, \rho=1$, and $N_{i}(t=0)=1$ for $i=1, \ldots, M$.

\subsection{Reminder: occupation probabilities and mean energy}

Taking box number 1 as a generic box, we denote by $f_{k}(t)$ the probability that it contains $k$ particles at time $t$ :

$$
f_{k}(t)=\operatorname{Prob}\left\{N_{1}(t)=k\right\} .
$$

The dynamical equations obeyed by the occupation probabilities $f_{k}(t)$ read [see e.g. Appendix A of ref. [23 for a derivation]

$$
\begin{aligned}
\frac{\mathrm{d} f_{k}(t)}{\mathrm{d} t} & =\frac{k+1}{\Lambda(t)} f_{k+1}(t)+f_{k-1}(t)-\left(1+\frac{k}{\Lambda(t)}\right) f_{k}(t) \quad(k \geq 2), \\
\frac{\mathrm{d} f_{1}(t)}{\mathrm{d} t} & =\frac{2}{\Lambda(t)} f_{2}(t)+\mu(t) f_{0}(t)-2 f_{1}(t), \\
\frac{\mathrm{d} f_{0}(t)}{\mathrm{d} t} & =f_{1}(t)-\mu(t) f_{0}(t),
\end{aligned}
$$

with the initial value

$$
f_{k}(0)=\delta_{k, 1}
$$

and where

$$
\frac{1}{\Lambda(t)}=1+\left(\mathrm{e}^{-\beta}-1\right) f_{0}(t), \quad \mu(t)=\mathrm{e}^{-\beta}+\left(1-\mathrm{e}^{-\beta}\right) f_{1}(t)
$$

Eqs. (2.4) describe a birth-death process, i.e., a random walk on the $k$-axis with position-dependent rates. The rate at which a ball enters a non-empty box $(k \geq 1)$ is equal to 1 , while it is equal to $\mu$ for an empty box. The first process is temperatureindependent as it should, since diffusion between non-empty boxes is not constrained by temperature. The second process depends on temperature. For instance, at infinite temperature, $\mu$ is equal to 1 , because moving a ball to an empty box is always allowed (diffusion and evaporation are both permitted). At zero temperature, $\mu$ is equal to $f_{1}$, which expresses the fact that moving a ball to an empty box is allowed if the departure box contains one ball only (only diffusion is permitted). The departure rate of a ball from a box containing $k$ balls is equal to $k / \Lambda(t)$. Hence, there is equilibration of $k$ around $\Lambda(t)$. At low temperature, $\Lambda(t) \approx\left(1-f_{0}(t)\right)^{-1}$, which is equal to the average number of balls in a non-empty box.

To summarize, eqs. (2.4) describe a random walk along the $k$-axis, in a confining potential centered around $\Lambda(t)$, which itself is slowly increasing, whenever the number 
of empty boxes increases [10]. It is indeed intuitively clear that while the equilibration inside the potential is fast, the evolution of $\Lambda(t)$ is very slow at low temperature. This defines two time scales, as mentioned in the Introduction, or two regimes, which are to be identified respectively with the $\beta$ - and the $\alpha$-regime, characteristic of glassy relaxation [9].

Eqs. (2.4) can be formally rewritten as a Markovian system, of the form

$$
\frac{\mathrm{d} f_{k}(t)}{\mathrm{d} t}=\sum_{\ell \geq 0} \mathcal{M}_{k \ell}\left[f_{0}(t), f_{1}(t)\right] f_{\ell}(t)
$$

where the matrix $\mathcal{M}\left[f_{0}(t), f_{1}(t)\right]$ satisfies

$$
\sum_{k \geq 0} \mathcal{M}_{k \ell}\left[f_{0}(t), f_{1}(t)\right]=0
$$

Actually, the reaction terms $\Lambda(t)$ and $\mu(t)$ involve $f_{0}(t)$ and $f_{1}(t)$, which makes the dynamics non-Markovian and the system of equations (2.4) or (2.7) non-linear.

Eqs. (2.4) or (2.7) ensure the conservation of the moments

$$
\sum_{k \geq 0} f_{k}(t)=1, \quad\left\langle N_{1}(t)\right\rangle=\sum_{k \geq 1} k f_{k}(t)=1
$$

respectively corresponding to the total numbers of boxes and of particles. Finally, the mean energy per box of the system reads $E(t)=-\left\langle\delta_{N_{1}(t), 0}\right\rangle=-f_{0}(t)$.

\subsection{Density correlation function}

The density correlation function $c(t, s)$ measures the correlation between the number of particles in box number 1 at times $s$ (waiting time) and $t$ (observation time), with $0 \leq s \leq t$ :

$$
c(t, s)=\left\langle N_{1}(t) N_{1}(s)\right\rangle-\left\langle N_{1}(t)\right\rangle\left\langle N_{1}(s)\right\rangle=\left\langle N_{1}(t) N_{1}(s)\right\rangle-1 .
$$

By definition, we have

$$
\left\langle N_{1}(t) N_{1}(s)\right\rangle=\sum_{j, k \geq 1} j k \operatorname{Prob}\left\{N_{1}(t)=k \mid N_{1}(s)=j\right\} \operatorname{Prob}\left\{N_{1}(s)=j\right\} .
$$

Eq. (2.10) can thus be rewritten as

$$
c(t, s)=\sum_{j, k \geq 1} j k f_{j}(s) g_{k}^{(j)}(t, s)-1,
$$

where

$$
g_{k}^{(j)}(t, s)=\operatorname{Prob}\left\{N_{1}(t)=k \mid N_{1}(s)=j\right\}
$$


or else as

$$
c(t, s)=\sum_{k \geq 1} k \gamma_{k}(t, s)-1,
$$

with

$$
\gamma_{k}(t, s)=\sum_{j \geq 1} j f_{j}(s) g_{k}^{(j)}(t, s) .
$$

Since the time variable $s$ plays the role of a parameter in the dynamics, both $g_{k}^{(j)}(t, s)$ and $\gamma_{k}(t, s)$ obey the dynamical equations

$$
\begin{aligned}
\frac{\partial g_{k}^{(j)}(t, s)}{\partial t} & =\sum_{\ell \geq 0} \mathcal{M}_{k \ell}\left[f_{0}(t), f_{1}(t)\right] g_{\ell}^{(j)}(t, s), \\
\frac{\partial \gamma_{k}(t, s)}{\partial t} & =\sum_{\ell \geq 0} \mathcal{M}_{k \ell}\left[f_{0}(t), f_{1}(t)\right] \gamma_{\ell}(t, s),
\end{aligned}
$$

with the initial conditions

$$
g_{k}^{(j)}(s, s)=\delta_{k, j}, \quad \gamma_{k}(s, s)=k f_{k}(s),
$$

implying in particular

$$
c(s, s)=\left\langle N_{1}(s)^{2}\right\rangle-1=\sum_{k \geq 1} k^{2} f_{k}(s)-1,
$$

in agreement with eq. (2.10). Eqs. (2.16, 2.17) ensure the conservation of probability, i.e.,

$$
\sum_{k \geq 0} g_{k}^{(j)}(t, s)=1, \quad \sum_{k \geq 0} \gamma_{k}(t, s)=1
$$

\subsection{Density response functions}

The local density response function $r(t, s)$ is a measure of the change in the density of box number 1 at time $t$, induced by an infinitesimal change in the conjugate variable, i.e., in the present case the local chemical potential acting on the same box, at the earlier time $s$. Assume that box number 1 is subjected to an arbitrary time-dependent chemical potential, given by $\alpha(t)$ in reduced units. The perturbed action now reads

$$
S(t)=-\beta \sum_{i=1}^{M} \delta_{N_{i}(t), 0}-\alpha(t) N_{1}(t) .
$$

The occupation probabilities of box number 1 now depend on $\alpha(t)$ : we denote them by $f_{k}^{\alpha}(t)=\operatorname{Prob}\left\{N_{1}(t)=k\right\}$. In the thermodynamical limit, i.e., to leading order as $M \rightarrow \infty$, the occupation probabilities of all the other boxes $(i=2, \ldots, M)$ are still 
given by the $f_{k}(t)$. Indeed the mean-field geometry of the model implies that the mutual influence of any two distinct boxes scales as $1 / M$.

Assuming for simplicity $|\alpha(t)|<\beta$, the $f_{k}^{\alpha}(t)$ obey the dynamical equations

$$
\begin{aligned}
& \frac{\mathrm{d} f_{k}^{\alpha}(t)}{\mathrm{d} t}=(k+1) \nu_{-}(t) f_{k+1}^{\alpha}(t)+\nu_{+}(t) f_{k-1}^{\alpha}(t)-\left(\nu_{+}(t)+k \nu_{-}(t)\right) f_{k}^{\alpha}(t) \quad(k \geq 2) \\
& \frac{\mathrm{d} f_{1}^{\alpha}(t)}{\mathrm{d} t}=2 \nu_{-}(t) f_{2}^{\alpha}(t)+\mu_{+}(t) f_{0}^{\alpha}(t)-\left(\nu_{+}(t)+\mu_{-}(t)\right) f_{1}^{\alpha}(t) \\
& \frac{\mathrm{d} f_{0}^{\alpha}(t)}{\mathrm{d} t}=\mu_{-}(t) f_{1}^{\alpha}(t)-\mu_{+}(t) f_{0}^{\alpha}(t)
\end{aligned}
$$

with the initial value

$$
f_{k}^{\alpha}(0)=\delta_{k, 1}
$$

and with the definitions

$$
\begin{aligned}
& \mu_{+}(t)=f_{1}(t) \mathcal{W}(-\alpha(t))+\left(1-f_{1}(t)\right) \mathrm{e}^{-\beta+\alpha(t)}, \\
& \mu_{-}(t)=1-f_{0}(t)+f_{0}(t) \mathcal{W}(\alpha(t)), \\
& \nu_{+}(t)=f_{1}(t)+\left(1-f_{1}(t)\right) \mathcal{W}(-\alpha(t)), \\
& \nu_{-}(t)=\left(1-f_{0}(t)\right) \mathcal{W}(\alpha(t))+f_{0}(t) \mathrm{e}^{-\beta-\alpha(t)} .
\end{aligned}
$$

These equations can be derived along the same lines as in ref. [23].

In the above expressions,

$$
\mathcal{W}(\Delta S)=\min \left(1, \mathrm{e}^{-\Delta S}\right)
$$

is the Metropolis acceptance rate associated with a change of action $\Delta S \equiv \beta \Delta E$. This rate is not differentiable at $\Delta S=0$. We have indeed

$$
\left.\frac{\mathrm{d} \mathcal{W}}{\mathrm{d} \Delta S}\right|_{\Delta S \rightarrow 0^{+}}=-1,\left.\quad \frac{\mathrm{d} \mathcal{W}}{\mathrm{d} \Delta S}\right|_{\Delta S \rightarrow 0^{-}}=0 .
$$

As a consequence, we are led to define the following two local response functions for the density

$$
r^{ \pm}(t, s)=\left.\frac{\delta\left\langle N_{1}(t)\right\rangle}{\delta \alpha(s)}\right|_{\alpha(s) \rightarrow 0^{ \pm}}
$$

Introducing the quantities

$$
h_{k}^{ \pm}(t, s)=\left.\frac{\delta f_{k}^{\alpha}(t)}{\delta \alpha(s)}\right|_{\alpha(s) \rightarrow 0^{ \pm}}
$$

we have

$$
r^{ \pm}(t, s)=\sum_{k \geq 1} k h_{k}^{ \pm}(t, s) .
$$


The dynamical equations for the $h_{k}^{ \pm}(t, s)$ are obtained by taking the functional derivative of eqs. (2.22) with respect to $\alpha(s)$ for $\alpha(s) \rightarrow 0^{ \pm}$. This yields

$$
\frac{\partial h_{k}^{ \pm}(t, s)}{\partial t}=\sum_{\ell \geq 0} \mathcal{M}_{k \ell}\left[f_{0}(t), f_{1}(t)\right] h_{\ell}^{ \pm}(t, s),
$$

with the initial conditions

$$
\begin{aligned}
h_{k}^{+}(s, s)= & -\frac{k+1}{\Lambda(s)} f_{k+1}(s)+\frac{k}{\Lambda(s)} f_{k}(s) \quad(k \geq 2), \\
h_{1}^{+}(s, s)= & -\frac{2}{\Lambda(s)} f_{2}(s)+\mu(s) f_{0}(s) \\
h_{0}^{+}(s, s)= & -\mu(s) f_{0}(s) \\
h_{k}^{-}(s, s)= & -(k+1) \mathrm{e}^{-\beta} f_{0}(s) f_{k+1}(s) \\
& +\left(1-f_{1}(s)\right) f_{k-1}(s)+\left(k \mathrm{e}^{-\beta} f_{0}(s)+f_{1}(s)-1\right) f_{k}(s) \quad(k \geq 2), \\
h_{1}^{-}(s, s)= & -2 \mathrm{e}^{-\beta} f_{0}(s) f_{2}(s)+\mu(s) f_{0}(s)+\left(f_{1}(s)-1\right) f_{1}(s) \\
h_{0}^{-}(s, s)= & -\mu(s) f_{0}(s) .
\end{aligned}
$$

We have

$$
\sum_{k \geq 0} h_{k}^{ \pm}(t, s)=0
$$

at all times $t \geq s$. Furthermore, eqs. (2.31) imply that both response functions coincide at initial times:

$$
r^{+}(s, s)=r^{-}(s, s)=1+\left(2 \mathrm{e}^{-\beta}-1\right) f_{0}(s)-f_{1}(s)+2\left(1-\mathrm{e}^{-\beta}\right) f_{0}(s) f_{1}(s) .
$$

\subsection{Fluctuation-dissipation ratios}

The fluctuation-dissipation ratio provides a measure of the violation of the fluctuationdissipation theorem, and thus of the departure of the system from equilibrium [9, 18, 22. In the present case, we need to define two fluctuation-dissipation ratios

$$
X^{ \pm}(t, s)=\frac{r^{ \pm}(t, s)}{\frac{\partial c(t, s)}{\partial s}}
$$

These definitions contain no explicit temperature dependence, because a factor of temperature has been absorbed in the definition of the reduced chemical potential $\alpha(t)$.

We are thus led to investigate the quantity

$$
\frac{\partial c(t, s)}{\partial s}=\sum_{k \geq 1} k \zeta_{k}(t, s)
$$


with

$$
\zeta_{k}(t, s)=\frac{\partial \gamma_{k}(t, s)}{\partial s}
$$

For $t>s$, the dynamical equations obeyed by these quantities are deduced from eq. (2.17) and read

$$
\frac{\partial \zeta_{k}(t, s)}{\partial t}=\sum_{\ell \geq 0} \mathcal{M}_{k \ell}\left[f_{0}(t), f_{1}(t)\right] \zeta_{\ell}(t, s) .
$$

Eq. (2.31) implies

$$
\sum_{k \geq 0} \zeta_{k}(t, s)=\frac{\partial}{\partial s} \sum_{k \geq 0} \gamma_{k}(t, s)=0 .
$$

Moreover, by integrating eq. (2.17) for the $\gamma_{k}(t, s)$ to first order in $\theta=t-s$, we obtain the initial values

$$
\zeta_{k}(s, s)=\sum_{\ell \geq 0}(k-\ell) \mathcal{M}_{k \ell}\left[f_{0}(t), f_{1}(t)\right] f_{\ell}(t)
$$

i.e.,

$$
\begin{aligned}
\zeta_{k}(s, s) & =-\frac{k+1}{\Lambda(s)} f_{k+1}(s)+f_{k-1}(s) \quad(k \geq 2) \\
\zeta_{1}(s, s) & =-\frac{2}{\Lambda(s)} f_{2}(s)+\mu(s) f_{0}(s), \\
\zeta_{0}(s, s) & =-f_{1}(s)
\end{aligned}
$$

implying

$$
\left.\frac{\partial c(t, s)}{\partial s}\right|_{t=s}=2-\frac{c(s, s)}{\Lambda(s)}+f_{0}(s)(\mu(s)-1) .
$$

\section{Generating functions and integral representations}

It is possible to obtain integral representations of the solutions of eqs. 2.4, 2.17, 2.30, 2.37) by means of the generating functions

$$
\begin{aligned}
F(x, t) & =\sum_{k \geq 0} f_{k}(t) x^{k}, \quad G(x, t, s)=\sum_{k \geq 0} \gamma_{k}(t, s) x^{k} \\
H^{ \pm}(x, t, s) & =\sum_{k \geq 0} h_{k}^{ \pm}(t, s) x^{k}, \quad Z(x, t, s)=\sum_{k \geq 0} \zeta_{k}(t, s) x^{k} .
\end{aligned}
$$

These functions obey the partial differential equations

$$
\begin{aligned}
\frac{\partial F(x, t)}{\partial t} & =(x-1)\left(F(x, t)-\frac{1}{\Lambda(t)} \frac{\partial F(x, t)}{\partial x}-Y_{f}(t)\right) \\
\frac{\partial G(x, t, s)}{\partial t} & =(x-1)\left(G(x, t, s)-\frac{1}{\Lambda(t)} \frac{\partial G(x, t, s)}{\partial x}-Y_{\gamma}(t, s)\right)
\end{aligned}
$$




$$
\begin{aligned}
\frac{\partial H^{ \pm}(x, t, s)}{\partial t} & =(x-1)\left(H^{ \pm}(x, t, s)-\frac{1}{\Lambda(t)} \frac{\partial H^{ \pm}(x, t, s)}{\partial x}-Y_{h^{ \pm}}(t, s)\right) \\
\frac{\partial Z(x, t, s)}{\partial t} & =(x-1)\left(Z(x, t, s)-\frac{1}{\Lambda(t)} \frac{\partial Z(x, t, s)}{\partial x}-Y_{\zeta}(t, s)\right) .
\end{aligned}
$$

The $Y$-functions which enter these equations read

$$
\begin{aligned}
Y_{f}(t) & =\left(1-\mathrm{e}^{-\beta}\right) f_{0}(t)=1-1 / \Lambda(t) \\
Y_{\gamma}(t, s) & =\left(1-\mathrm{e}^{-\beta}\right)\left(\gamma_{0}(t, s)+f_{0}(t) \frac{\partial \gamma_{0}(t, s)}{\partial t}-\frac{\mathrm{d} f_{0}(t)}{\mathrm{d} t} \gamma_{0}(t, s)\right), \\
Y_{h^{ \pm}}(t, s) & =\left(1-\mathrm{e}^{-\beta}\right)\left(h_{0}^{ \pm}(t, s)+f_{0}(t) \frac{\partial h_{0}^{ \pm}(t, s)}{\partial t}-\frac{\mathrm{d} f_{0}(t)}{\mathrm{d} t} h_{0}^{ \pm}(t, s)\right), \\
Y_{\zeta}(t, s) & =\left(1-\mathrm{e}^{-\beta}\right)\left(\zeta_{0}(t, s)+f_{0}(t) \frac{\partial \zeta_{0}(t, s)}{\partial t}-\frac{\mathrm{d} f_{0}(t)}{\mathrm{d} t} \zeta_{0}(t, s)\right) .
\end{aligned}
$$

The initial conditions for eq. (3.2) are derived from eqs. (2.5, 2.18, 2.31, 2.40):

$$
\begin{aligned}
F(x, 0) & =x, \\
G(x, s, s) & =x \frac{\partial F(x, s)}{\partial x}, \\
H^{+}(x, s, s) & =(x-1)\left(\frac{1}{\Lambda(s)} \frac{\partial F(x, s)}{\partial x}+\mathrm{e}^{-\beta} f_{0}(s)-f_{1}(s)+2\left(1-\mathrm{e}^{-\beta}\right) f_{0}(s) f_{1}(s)\right), \\
H^{-}(x, s, s) & =(x-1) \\
& \times\left(\left(1-f_{1}(s)\right) F(x, s)+\mathrm{e}^{-\beta} f_{0}(s) \frac{\partial F(x, s)}{\partial x}+\left(1-\mathrm{e}^{-\beta}\right) f_{0}(s)\left(2 f_{1}(s)-1\right)\right), \\
Z(x, s, s) & =-\frac{1}{\Lambda(s)} \frac{\partial F(x, s)}{\partial x}+x F(x, s)-\left(1-\mathrm{e}^{-\beta}\right) f_{0}(s)\left((1-x) f_{1}(s)+x\right) .
\end{aligned}
$$

The conservation of moments expressed by eqs. (2.9, 2.20, 2.32, 2.38) implies

$$
F(1, t)=\left.\frac{\partial F(x, t)}{\partial x}\right|_{x=1}=G(1, t, s)=1, \quad H^{ \pm}(1, t, s)=Z(1, t, s)=0 .
$$

Eqs. (3.2) can be solved by the method of characteristics [see e.g. Appendix B of ref. [23]. We thus obtain

$$
\begin{aligned}
F(x, t) & =\left(1+(x-1) \mathrm{e}^{-\tau(t)}\right) \mathrm{e}^{(x-1) D(t, 0)}+\int_{0}^{t} \mathrm{~d} u \mathcal{K}(x, t, u) Y_{f}(u), \\
G(x, t, s) & =\mathrm{e}^{(x-1) D(t, s)} G\left(1+(x-1) \mathrm{e}^{\tau(s)-\tau(t)}, s, s\right)+\int_{s}^{t} \mathrm{~d} u \mathcal{K}(x, t, u) Y_{\gamma}(u, s),
\end{aligned}
$$

and expressions for $H^{ \pm}(x, t, s)$ and $Z(x, t, s)$ similar to that for $G(x, t, s)$, with the definitions

$$
\tau(t)=\int_{0}^{t} \frac{\mathrm{d} u}{\Lambda(u)},
$$




$$
\begin{aligned}
D(t, u) & =\int_{u}^{t} \mathrm{~d} v \mathrm{e}^{\tau(v)-\tau(t)} \\
\mathcal{K}(x, t, u) & =(1-x) \mathrm{e}^{\tau(u)-\tau(t)+(x-1) D(t, u)} .
\end{aligned}
$$

The quantities of interest are given by

$$
c(t, s)=\left.\frac{\partial G(x, t, s)}{\partial x}\right|_{x=1}-1, r^{ \pm}(t, s)=\left.\frac{\partial H^{ \pm}(x, t, s)}{\partial x}\right|_{x=1}, \frac{\partial c(t, s)}{\partial s}=\left.\frac{\partial Z(x, t, s)}{\partial x}\right|_{x=1} .
$$

Using eqs. (3.6) and the corresponding equations for $H^{ \pm}(x, t, s)$ and $Z(x, t, s)$, we obtain finally

$$
\begin{aligned}
c(t, s) & =D(t, s)-1+(c(s, s)+1) \mathrm{e}^{\tau(s)-\tau(t)}-\int_{s}^{t} \mathrm{~d} u \mathrm{e}^{\tau(u)-\tau(t)} Y_{\gamma}(u, s), \\
r^{ \pm}(t, s) & =r^{ \pm}(s, s) \mathrm{e}^{\tau(s)-\tau(t)}-\int_{s}^{t} \mathrm{~d} u \mathrm{e}^{\tau(u)-\tau(t)} Y_{h^{ \pm}}(u, s), \\
\frac{\partial c(t, s)}{\partial s} & =\frac{\partial c(s, s)}{\partial s} \mathrm{e}^{\tau(s)-\tau(t)}-\int_{s}^{t} \mathrm{~d} u \mathrm{e}^{\tau(u)-\tau(t)} Y_{\zeta}(u, s) .
\end{aligned}
$$

Setting $x=0$ in eqs. (3.6), we get integral representations of the following quantities, which will be useful in Sec. 6:

$$
\begin{aligned}
f_{0}(t) & =\left(1-\mathrm{e}^{-\tau(t)}\right) \mathrm{e}^{-D(t, 0)}+\int_{0}^{t} \mathrm{~d} u \mathcal{K}(0, t, u) Y_{f}(u), \\
\gamma_{0}(t, s) & =\mathrm{e}^{-D(t, s)} G\left(1-\mathrm{e}^{\tau(s)-\tau(t)}, s, s\right)+\int_{s}^{t} \mathrm{~d} u \mathcal{K}(0, t, u) Y_{\gamma}(u, s),
\end{aligned}
$$

with

$$
\mathcal{K}(0, t, u)=\mathrm{e}^{\tau(u)-\tau(t)-D(t, u)}=\frac{\partial}{\partial u} \mathrm{e}^{-D(t, u)},
$$

and similarly

$$
\begin{aligned}
h_{0}^{ \pm}(t, s) & =\mathrm{e}^{-D(t, s)} H^{ \pm}\left(1-\mathrm{e}^{\tau(s)-\tau(t)}, s, s\right)+\int_{s}^{t} \mathrm{~d} u \mathcal{K}(0, t, u) Y_{h^{ \pm}}(u, s), \\
\zeta_{0}(t, s) & =\mathrm{e}^{-D(t, s)} Z\left(1-\mathrm{e}^{\tau(s)-\tau(t)}, s, s\right)+\int_{s}^{t} \mathrm{~d} u \mathcal{K}(0, t, u) Y_{\zeta}(u, s) .
\end{aligned}
$$

\section{Infinite-temperature behavior}

At infinite temperature, the Backgammon model, which is a 'thermodynamical' generalization of the Ehrenfest model, as discussed in the Introduction, is solvable. The solutions given in eqs. (3.6, 3.11) indeed become explicit, because of the simplifications $\mathrm{e}^{-\beta}=\Lambda(t)=\mu(t)=1$. 


\subsection{Reminder: occupation probabilities and mean energy}

Since $Y_{f}(t)=0$, we have

$$
F(x, t)=\left(1+(x-1) \mathrm{e}^{-t}\right) \mathrm{e}^{(x-1)\left(1-\mathrm{e}^{-t}\right)} .
$$

The occupation probabilities thus read

$$
f_{k}(t)=\left(\left(1-\mathrm{e}^{-t}\right)^{2}+k \mathrm{e}^{-t}\right) \frac{\left(1-\mathrm{e}^{-t}\right)^{k-1} \mathrm{e}^{\mathrm{e}^{-t}-1}}{k !},
$$

yielding, in particular, the mean energy

$$
E(t)=-f_{0}(t)=-\left(1-\mathrm{e}^{-t}\right) \mathrm{e}^{\mathrm{e}^{-t}-1}
$$

\subsection{Correlation and response, fluctuation-dissipation ratios}

Similarly, noticing that $Y_{\gamma}(t, s)=Y_{h^{ \pm}}(t, s)=Y_{\zeta}(t, s)=0$, we get

$$
\begin{aligned}
G(x, t, s) & =\left(1+(x-1) \mathrm{e}^{s-t}\right)\left(1+(x-1) \mathrm{e}^{-t}\left(1-\mathrm{e}^{-s}\right)\right) \mathrm{e}^{(x-1)\left(1-\mathrm{e}^{-t}\right)}, \\
H^{+}(x, t, s) & =(x-1) \\
& \times\left[\left(\mathrm{e}^{s}+(x-1) \mathrm{e}^{-t}\left(\mathrm{e}^{-s}-1\right)\right) \mathrm{e}^{(x-1)\left(1-\mathrm{e}^{-t}\right)-t}-\mathrm{e}^{(x-1)\left(1-\mathrm{e}^{s-t}\right)-s-t+\mathrm{e}^{-s}-1}\right], \\
H^{-}(x, t, s) & =(x-1) \mathrm{e}^{(x-1)\left(1-\mathrm{e}^{-t}\right)-t} \\
& \times\left[\left(1+(x-1) \mathrm{e}^{-t}\right) \mathrm{e}^{(x-1) s}-\left(1+(x-1) \mathrm{e}^{s-t}\right) \mathrm{e}^{-s+\mathrm{e}^{-s}-1}\right] \\
Z(x, t, s) & =(x-1)\left((x-1) \mathrm{e}^{-t}+1+\mathrm{e}^{-2 s}\right) \mathrm{e}^{(x-1)\left(1-\mathrm{e}^{-t}\right)+s-t}
\end{aligned}
$$

We thus have

$$
c(t, s)=\left(1-\mathrm{e}^{-2 s}\right) \mathrm{e}^{s-t}, \quad r^{ \pm}(t, s)=\left(1-\mathrm{e}^{-2 s+\mathrm{e}^{-s}-1}\right) \mathrm{e}^{s-t}, \quad \frac{\partial c(t, s)}{\partial s}=\left(1+\mathrm{e}^{-2 s}\right) \mathrm{e}^{s-t} .
$$

Note that both response functions coincide for all times at infinite temperature: $r^{+}(t, s)=$

$r^{-}(t, s)$, whereas the generating functions $H^{ \pm}(x, t, s)$ are different from each other. The common value of the fluctuation-dissipation ratios is independent of $t$, and reads

$$
X^{ \pm}(t, s)=\frac{1-\mathrm{e}^{-2 s+\mathrm{e}^{-s}-1}}{1+\mathrm{e}^{-2 s}}
$$

At equilibrium, i.e., for $s \gg 1$, the correlation and response functions only depend on the time difference $\theta=t-s$, and they fulfil the fluctuation-dissipation theorem (see Sec. 5):

$$
c_{\mathrm{eq}}(\theta)=r_{\mathrm{eq}}^{ \pm}(\theta)=-\frac{\mathrm{d} c_{\mathrm{eq}}(\theta)}{\mathrm{d} \theta}=\mathrm{e}^{-\theta} .
$$

Equivalently, $X^{ \pm}(t, s)$ in eq. (4.6) goes to unity as $s \rightarrow \infty$. 


\section{$5 \quad$ Equilibrium properties}

\subsection{Reminder: occupation probabilities and mean energy}

At any finite temperature and for long enough times, the system converges towards thermal equilibrium. The occupation probabilities at equilibrium are given by the stationary solution of the dynamical equations (2.4):

$$
\begin{aligned}
\left(f_{k}\right)_{\mathrm{eq}} & =\mathrm{e}^{-\Lambda_{\mathrm{eq}}} \frac{\Lambda_{\mathrm{eq}}^{k-1}}{k !} \quad(k \geq 1), \\
-E_{\mathrm{eq}} & =\left(f_{0}\right)_{\mathrm{eq}}=\frac{\Lambda_{\mathrm{eq}}-1+\mathrm{e}^{-\Lambda_{\mathrm{eq}}}}{\Lambda_{\mathrm{eq}}}=\frac{\mathrm{e}^{\beta-\Lambda_{\mathrm{eq}}}}{\Lambda_{\mathrm{eq}}}
\end{aligned}
$$

where $\Lambda_{\text {eq }}$, the equilibrium value of $\Lambda(t)$, is related to temperature by

$$
\mathrm{e}^{\beta}=1+\left(\Lambda_{\mathrm{eq}}-1\right) \mathrm{e}^{\Lambda_{\mathrm{eq}}}
$$

This quantity can be identified with the thermodynamical fugacity of the model [see Appendix $\mathrm{C}$ of ref. [23]]. Note that the most probable non-zero occupancy is approximately equal to $\Lambda_{\text {eq }}$, in agreement with the discussion given below eq. (2.6).

At low temperature, we have

$$
\begin{aligned}
& \Lambda_{\mathrm{eq}}=\beta-\ln \beta+\frac{\ln \beta+1}{\beta}+\frac{\ln ^{2} \beta-1}{2 \beta^{2}}+\cdots, \\
& E_{\mathrm{eq}}=-1+\frac{1}{\beta}+\frac{\ln \beta}{\beta^{2}}+\frac{\ln ^{2} \beta-\ln \beta-1}{\beta^{3}}+\cdots
\end{aligned}
$$

Finally, the generating function of the equilibrium occupation probabilities reads

$$
F_{\mathrm{eq}}(x)=\frac{\Lambda_{\mathrm{eq}}-1+\mathrm{e}^{(x-1) \Lambda_{\mathrm{eq}}}}{\Lambda_{\mathrm{eq}}} .
$$

The convergence towards equilibrium is characterized by a relaxation time $\tilde{t}_{\mathrm{eq}}$ (denoted as $t_{\mathrm{eq}}^{(1)}$ in ref. [23]), which is exponentially divergent at low temperature [see eq. (6.9)].

\subsection{Density correlation and response functions}

At equilibrium, the density correlation function $c(t, s)=c_{\mathrm{eq}}(\theta)$ and response functions $r^{ \pm}(t, s)=r_{\mathrm{eq}}^{ \pm}(\theta)$ are stationary: they only depend on the time difference $\theta=t-s$.

These quantities can be derived from eqs. (3.11), where all integrals become convolutions. These equations can therefore be solved in Laplace space. Denoting by $\hat{c}_{\mathrm{eq}}(p)$ 
the Laplace transform of $c_{\mathrm{eq}}(\theta)$, and using similar conventions for the other functions, we obtain after some algebra

$$
\hat{r}_{\mathrm{eq}}^{+}(p)=\hat{r}_{\mathrm{eq}}^{-}(p)=\Lambda_{\mathrm{eq}}-p \hat{c}_{\mathrm{eq}}(p)
$$

which express that both density response functions coincide at equilibrium, and that they obey the identity

$$
r_{\mathrm{eq}}^{ \pm}(\theta)=-\frac{\mathrm{d} c_{\mathrm{eq}}(\theta)}{\mathrm{d} \theta}
$$

In other words, the fluctuation-dissipation ratios $X^{ \pm}(\theta)$ are identically equal to unity at equilibrium, expressing thus the fluctuation-dissipation theorem.

Furthermore, we obtain the explicit results

$$
\hat{r}_{\text {eq }}^{ \pm}(p)=\frac{\Lambda_{\text {eq }}-\left(\Lambda_{\text {eq }}-1\right)\left(p+1 /\left(f_{0}\right)_{\text {eq }}\right)\left(\hat{K}\left(p, \Lambda_{\text {eq }}\right)-\frac{\Lambda_{\text {eq }} \mathrm{e}^{-\Lambda_{\text {eq }}}}{1+p \Lambda_{\text {eq }}}\right)}{\left(1+p \Lambda_{\text {eq }}\right)\left(\Lambda_{\text {eq }}-\left(\Lambda_{\text {eq }}-1\right)\left(p+1 /\left(f_{0}\right)_{\text {eq }}\right) \hat{K}\left(p, \Lambda_{\text {eq }}\right)\right)},
$$

where

$$
\hat{K}\left(p, \Lambda_{\text {eq }}\right)=\Lambda_{\text {eq }} \int_{0}^{1} \mathrm{~d} z z^{p \Lambda_{\text {eq }}} \mathrm{e}^{\Lambda_{\text {eq }}(z-1)}=\mathrm{e}^{-\Lambda_{\text {eq }}} \sum_{k \geq 0} \frac{1}{p+k / \Lambda_{\text {eq }}} \frac{\Lambda_{\text {eq }}^{k-1}}{(k-1) !}
$$

is the Laplace transform of the equilibrium kernel

$$
K\left(\theta, \Lambda_{\mathrm{eq}}\right)=\exp \left(-\theta / \Lambda_{\mathrm{eq}}-\Lambda_{\mathrm{eq}}\left(1-\mathrm{e}^{-\theta / \Lambda_{\mathrm{eq}}}\right)\right)
$$

The expression (5.7) is a meromorphic function, whose denominator coincides with that found in ref. [23] in the case of the energy correlation and response functions. This denominator has an infinite sequence of zeros on the real negative axis, which we denote by $p=-p_{k}^{(2)}$, with $k \geq 1$. The relaxation time of the equilibrium correlation and response functions $t_{\mathrm{eq}}$ (denoted as $t_{\mathrm{eq}}^{(2)}$ in ref. [23]) is given by the inverse of the smallest one:

$$
t_{\mathrm{eq}}=\frac{1}{p_{1}^{(2)}} \text {. }
$$

At low temperature, $t_{\text {eq }}$ is exponentially divergent ( $\alpha$-relaxation), while all the other characteristic times remain microscopic, i.e., of order $\Lambda_{\mathrm{eq}}$ ( $\beta$-relaxation). Indeed, we have

$$
\begin{aligned}
t_{\mathrm{eq}} & \approx \frac{\left(\Lambda_{\mathrm{eq}}-1\right) \mathrm{e}^{\Lambda_{\mathrm{eq}}}}{\Lambda_{\mathrm{eq}}^{2}}\left(\Lambda_{\mathrm{eq}}^{2} \mathrm{e}^{-\Lambda_{\mathrm{eq}}} I\left(\Lambda_{\mathrm{eq}}\right)+1-\Lambda_{\mathrm{eq}}\right) \\
& \approx \frac{2 \mathrm{e}^{\Lambda_{\mathrm{eq}}}}{\Lambda_{\mathrm{eq}}}\left(1+\frac{2}{\Lambda_{\mathrm{eq}}^{2}}+\cdots\right) \approx \frac{2 \mathrm{e}^{\beta}}{\beta^{2}}\left(1+\frac{2 \ln \beta+1}{\beta}+\cdots\right)
\end{aligned}
$$


with

$$
I(\Lambda)=\int_{0}^{1} \frac{\mathrm{d} z}{z}\left(\mathrm{e}^{\Lambda z}-1\right)=\sum_{n \geq 1} \frac{\Lambda^{n}}{n n !} \approx \frac{\mathrm{e}^{\Lambda}}{\Lambda} \sum_{\ell \geq 0} \frac{\ell !}{\Lambda^{\ell}} .
$$

The density correlation and response functions read

$$
r_{\mathrm{eq}}^{ \pm}(\theta)=\sum_{k \geq 1} A_{k} \mathrm{e}^{-p_{k}^{(2)} \theta}, \quad c_{\mathrm{eq}}(\theta)=\sum_{k \geq 1} \frac{A_{k}}{p_{k}^{(2)}} \mathrm{e}^{-p_{k}^{(2)} \theta},
$$

where the $A_{k}$ are the residues of the expression (5.7) at the poles $p=-p_{k}^{(2)}$. In particular the values of these functions at equal times $(\theta=0)$ provide the following sum rules for the residues $A_{k}$ :

$$
r_{\mathrm{eq}}^{ \pm}(0)=\sum_{k \geq 1} A_{k}=\frac{1+\left(\Lambda_{\mathrm{eq}}-1\right) \mathrm{e}^{-\Lambda_{\mathrm{eq}}}}{\Lambda_{\mathrm{eq}}}, \quad c_{\mathrm{eq}}(0)=\sum_{k \geq 1} \frac{A_{k}}{p_{k}^{(2)}}=\Lambda_{\mathrm{eq}} .
$$

The only difference between the expressions (5.13) of the density correlation and response functions at equilibrium and those for the energy [23] lies in the values of the residues: $A_{k}$ for the density and $a_{k}$ for the energy, with

$$
\frac{A_{k}}{a_{k}}=\left(\frac{\left(\Lambda_{\mathrm{eq}}-1\right)\left(p_{k}^{(2)}-1 /\left(f_{0}\right)_{\mathrm{eq}}\right)}{\Lambda_{\mathrm{eq}} p_{k}^{(2)}-1}\right)^{2} .
$$

Finally, the analysis presented in this section leads to the following description of the behavior of the equilibrium density correlation and response functions at low temperature.

- In the $\beta$-regime $(\theta \sim 1)$, expression (5.7) simplifies to

$$
\hat{r}_{\mathrm{eq}}^{ \pm}(p)=\Lambda_{\mathrm{eq}}-p \hat{c}_{\mathrm{eq}}(p) \approx \frac{1}{1+p \Lambda_{\mathrm{eq}}},
$$

up to an exponentially small correction. We thus obtain

$$
r_{\mathrm{eq}}^{ \pm}(\theta) \approx \frac{\mathrm{e}^{-\theta / \Lambda_{\mathrm{eq}}}}{\Lambda_{\mathrm{eq}}}, \quad c_{\mathrm{eq}}(\theta) \approx \Lambda_{\mathrm{eq}}-1+\mathrm{e}^{-\theta / \Lambda_{\mathrm{eq}}}
$$

- In the $\alpha$-regime $\left(\theta \sim t_{\mathrm{eq}}\right)$, the expressions (5.13) are dominated by the first term $(k=1)$. The corresponding residue can be estimated as $A_{1} \approx\left(\Lambda_{\mathrm{eq}}-1\right) / t_{\mathrm{eq}}$. We thus have

$$
r_{\mathrm{eq}}^{ \pm}(\theta) \approx \frac{\Lambda_{\mathrm{eq}}-1}{t_{\mathrm{eq}}} \mathrm{e}^{-\theta / t_{\mathrm{eq}}}, \quad c_{\mathrm{eq}}(\theta) \approx\left(\Lambda_{\mathrm{eq}}-1\right) \mathrm{e}^{-\theta / t_{\mathrm{eq}}} .
$$

- In the crossover between both regimes $\left(1 \ll \theta \ll t_{\text {eq }}\right)$, the density correlation and response functions exhibit the plateau values

$$
\left(r_{\mathrm{eq}}^{ \pm}\right)_{\mathrm{pl}} \approx \frac{\Lambda_{\mathrm{eq}}-1}{t_{\mathrm{eq}}}, \quad\left(c_{\mathrm{eq}}\right)_{\mathrm{pl}} \approx \Lambda_{\mathrm{eq}}-1 .
$$




\section{Non-equilibrium behavior at low temperature}

In this section, we shall extend to density fluctuations the low-temperature analysis developed in refs. [21, 23. This analytical approach is valid at low temperature, throughout the non-equilibrium $\alpha$-regime $(s \gg 1, t-s \gg 1)$, irrespective of the relative values of $s$ or $t$ with respect to the relaxation time $t_{\mathrm{eq}}$.

\subsection{Reminder: mean energy}

In the case of the mean energy, our approach consists in simplifying the first integral equation of eq. (3.12), by expanding the integrand as a power series in $t-u$. Keeping consistently the first two terms of this expansion, we are left with the following differential equation

$$
f_{0}(t) \approx \mathrm{e}^{-\Lambda(t)}+J_{0}(t) Y_{f}(t)+J_{1}(t) \frac{\mathrm{d} Y_{f}(t)}{\mathrm{d} t}
$$

with

$$
\begin{aligned}
& J_{0}(t)=\int_{0}^{\infty} \mathrm{d} \varepsilon K(\varepsilon, \Lambda(t))=\hat{K}(0, \Lambda(t))=1-\mathrm{e}^{-\Lambda(t)}, \\
& J_{1}(t)=-\int_{0}^{\infty} \mathrm{d} \varepsilon \varepsilon K(\varepsilon, \Lambda(t))=\left.\frac{\mathrm{d} \hat{K}(p, \Lambda(t))}{\mathrm{d} p}\right|_{p=0}=-\Lambda(t) \mathrm{e}^{-\Lambda(t)} I(\Lambda(t)) .
\end{aligned}
$$

This approach leads to the evolution equation [23]

$$
\frac{\mathrm{d} \Lambda}{\mathrm{d} t} \approx A\left(\Lambda(t), \Lambda_{\mathrm{eq}}\right)
$$

with

$$
A\left(\Lambda, \Lambda_{\mathrm{eq}}\right)=\frac{1}{I(\Lambda)}\left(1-\frac{(\Lambda-1) \mathrm{e}^{\Lambda}}{\left(\Lambda_{\mathrm{eq}}-1\right) \mathrm{e}^{\Lambda_{\mathrm{eq}}}}\right) .
$$

Eq. (6.3) describes the relaxation of energy throughout the $\alpha$-regime at low temperature. In particular,

- At zero temperature, and more generally in the aging regime $\left(1 \ll t \ll t_{\text {eq }}\right)$, we have

$$
\frac{\mathrm{d} \Lambda}{\mathrm{d} t} \approx \frac{1}{I(\Lambda)}
$$

hence

$$
t \approx \int_{0}^{\Lambda} \mathrm{d} \Lambda^{\prime} I\left(\Lambda^{\prime}\right)=\int_{0}^{1} \frac{\mathrm{d} z}{z^{2}}\left(\mathrm{e}^{\Lambda z}-1-\Lambda z\right)=\sum_{n \geq 1} \frac{\Lambda^{n+1}}{n(n+1) !} \approx \frac{\mathrm{e}^{\Lambda}}{\Lambda} \sum_{\ell \geq 0} \frac{(\ell+1) !}{\Lambda^{\ell}}
$$

or else

$$
\Lambda(t) \approx \ln t+\ln \ln t+\frac{\ln \ln t-2}{\ln t}+\cdots
$$


- In the opposite limit $\left(t \gg t_{\mathrm{eq}}\right)$, the convergence of $\Lambda(t)$ towards its equilibrium value $\Lambda_{\text {eq }}$ is exponential,

$$
\Lambda_{\mathrm{eq}}-\Lambda(t) \sim \mathrm{e}^{-t / \tilde{t}_{\mathrm{eq}}}
$$

with a relaxation time

$$
\begin{aligned}
\tilde{t}_{\mathrm{eq}} & \approx \frac{\Lambda_{\mathrm{eq}}-1}{\Lambda_{\mathrm{eq}}} I\left(\Lambda_{\mathrm{eq}}\right) \\
& \approx \frac{\mathrm{e}^{\Lambda_{\mathrm{eq}}}}{\Lambda_{\mathrm{eq}}}\left(1+\frac{1}{\Lambda_{\mathrm{eq}}^{2}}+\cdots\right) \approx \frac{\mathrm{e}^{\beta}}{\beta^{2}}\left(1+\frac{2 \ln \beta+1}{\beta}+\cdots\right)
\end{aligned}
$$

which is roughly equal to half the relaxation time $t_{\mathrm{eq}}$ of the correlation and response functions, given in eq. (5.11).

\subsection{Correlation and response, fluctuation-dissipation ratios}

The low-temperature approach consists in simplifying the integral equations (3.12, 3.14) for $\gamma_{0}(t, s), h_{0}^{ \pm}(t, s)$, and $\zeta_{0}(t, s)$ into the differential equations

$$
\begin{aligned}
\gamma_{0}(t, s) & \approx \mathrm{e}^{-\Lambda(t)}+J_{0}(t, s) Y_{\gamma}(t, s)+J_{1}(t, s) \frac{\partial Y_{\gamma}(t, s)}{\partial t} \\
h_{0}^{ \pm}(t, s) & \approx J_{0}(t, s) Y_{h^{ \pm}}(t, s)+J_{1}(t, s) \frac{\partial Y_{h^{ \pm}}(t, s)}{\partial t} \\
\zeta_{0}(t, s) & \approx J_{0}(t, s) Y_{\zeta}(t, s)+J_{1}(t, s) \frac{\partial Y_{\zeta}(t, s)}{\partial t} .
\end{aligned}
$$

These expressions lead, after some algebra, to the differential evolution equation

$$
\frac{1}{\phi(t, s)} \frac{\partial \phi(t, s)}{\partial t} \approx-B\left(\Lambda(t), \Lambda_{\mathrm{eq}}\right)
$$

where $\phi(t, s)$ denotes either $\gamma_{0}(t, s)-f_{0}(t), h_{0}^{ \pm}(t, s)$, or $\zeta_{0}(t, s)$, and with

$$
B\left(\Lambda, \Lambda_{\mathrm{eq}}\right)=\frac{A\left(\Lambda, \Lambda_{\mathrm{eq}}\right)+\Lambda^{2}\left(\mathrm{e}^{-\Lambda}+\frac{\mathrm{e}^{-\Lambda_{\mathrm{eq}}}}{\Lambda_{\mathrm{eq}}-1}\right)}{\Lambda\left(\Lambda^{2} \mathrm{e}^{-\Lambda} I(\Lambda)+1-\Lambda\right)} .
$$

Changing time variables from $s$ and $t$ to $\Lambda(s)$ and $\Lambda(t)$, we obtain the alternative form

$$
\frac{1}{\phi(t, s)} \frac{\partial \phi(t, s)}{\partial \Lambda(t)} \approx-\alpha\left(\Lambda(t), \Lambda_{\mathrm{eq}}\right)
$$

with

$$
\alpha\left(\Lambda, \Lambda_{\mathrm{eq}}\right)=\frac{B\left(\Lambda, \Lambda_{\mathrm{eq}}\right)}{A\left(\Lambda, \Lambda_{\mathrm{eq}}\right)} .
$$


The normalized solution of the differential equation (6.13) reads

$$
\phi(t, s) \approx \exp \left(-\int_{\Lambda(s)}^{\Lambda(t)} \mathrm{d} \Lambda^{\prime} \alpha\left(\Lambda^{\prime}, \Lambda_{\mathrm{eq}}\right)\right) .
$$

This result can be recast into a multiplicative scaling law

$$
\phi(t, s) \approx \frac{\Phi\left(\Lambda(s), \Lambda_{\mathrm{eq}}\right)}{\Phi\left(\Lambda(t), \Lambda_{\mathrm{eq}}\right)},
$$

with

$$
\Phi\left(\Lambda, \Lambda_{\mathrm{eq}}\right)=\exp \left(\int_{1}^{\Lambda} \mathrm{d} \Lambda^{\prime} \alpha\left(\Lambda^{\prime}, \Lambda_{\mathrm{eq}}\right)\right) .
$$

Finally, using eqs. (3.11) and the definitions of the $Y$-functions, we obtain the scaling predictions

$$
\frac{c(t, s)}{c_{\mathrm{pl}}(s)} \approx \frac{r^{ \pm}(t, s)}{r_{\mathrm{pl}}^{ \pm}(s)} \approx \frac{\partial c(t, s) / \partial s}{(\partial c / \partial s)_{\mathrm{pl}}(s)} \approx \frac{\Lambda(t)}{\Lambda(s)} \frac{\Phi\left(\Lambda(s), \Lambda_{\mathrm{eq}}\right)}{\Phi\left(\Lambda(t), \Lambda_{\mathrm{eq}}\right)}
$$

throughout the non-equilibrium $\alpha$-regime, which constitute the main result of this section. The plateau values $c_{\mathrm{pl}}(s), r_{\mathrm{pl}}^{ \pm}(s)$, and $(\partial c / \partial s)_{\mathrm{pl}}(s)$ in eq. (6.18) are the initial conditions for the dynamical equations (6.11, 6.13). These plateau values generalize the results (5.19) to the generic non-equilibrium situation at low temperature. They are attained when the fast $(\beta)$ modes are extinct, i.e., for $1 \ll \theta=t-s \ll s$. These quantities, which are non-trivial in general, remain to be determined, since their evaluation does not pertain to the analysis of the $\alpha$-regime stricto sensu.

In the case of the correlation function $c(t, s)$, the plateau value

$$
c_{\mathrm{pl}}(s)=\Lambda(s)-1
$$

is simply obtained by replacing $\Lambda_{\text {eq }}$ by $\Lambda(s)$ in the expression (5.19) at equilibrium. More generally, we have

$$
c(t, s) \approx c_{\mathrm{eq}}(t-s ; \Lambda(s)) \approx \Lambda(s)-1+\mathrm{e}^{-(t-s) / \Lambda(s)}
$$

[see eq. 5.18)] in the non-equilibrium $\beta$-regime. This expression amounts to saying that the system is somehow at an instantaneous equilibrium described by $\Lambda(s)$. This simple description is only justified for moderate times $(t-s \ll s)$, and for quantities, such as $c(t, s)$, which remain of order unity for long waiting times and/or at low temperature.

Figure 1 illustrates the validity of the above description. The zero-temperature correlation function $c(t, s)$ is plotted against $\ln (t-s)$, for different values of the waiting time $s$, indicated on the curves. The full lines show the result of a direct numerical integration of eqs. (2.4, 2.17). The dashed lines show (to the left, in the $\beta$-regime) eq. (6.20) and 
(to the right, in the $\alpha$-regime) the scaling law (6.18), with the plateau value (6.19). The quantitative agreement between the exact numerical data and their analytical description is increasingly convincing as the waiting time $s$ increases.

The evaluation of the other plateau values, namely $(\partial c / \partial s)_{\mathrm{pl}}(s)$ and $r_{\mathrm{pl}}^{ \pm}(s)$, requires more care, since these quantities are already exponentially small at equilibrium. A sketch of the method used for their derivation is given in Appendix A.

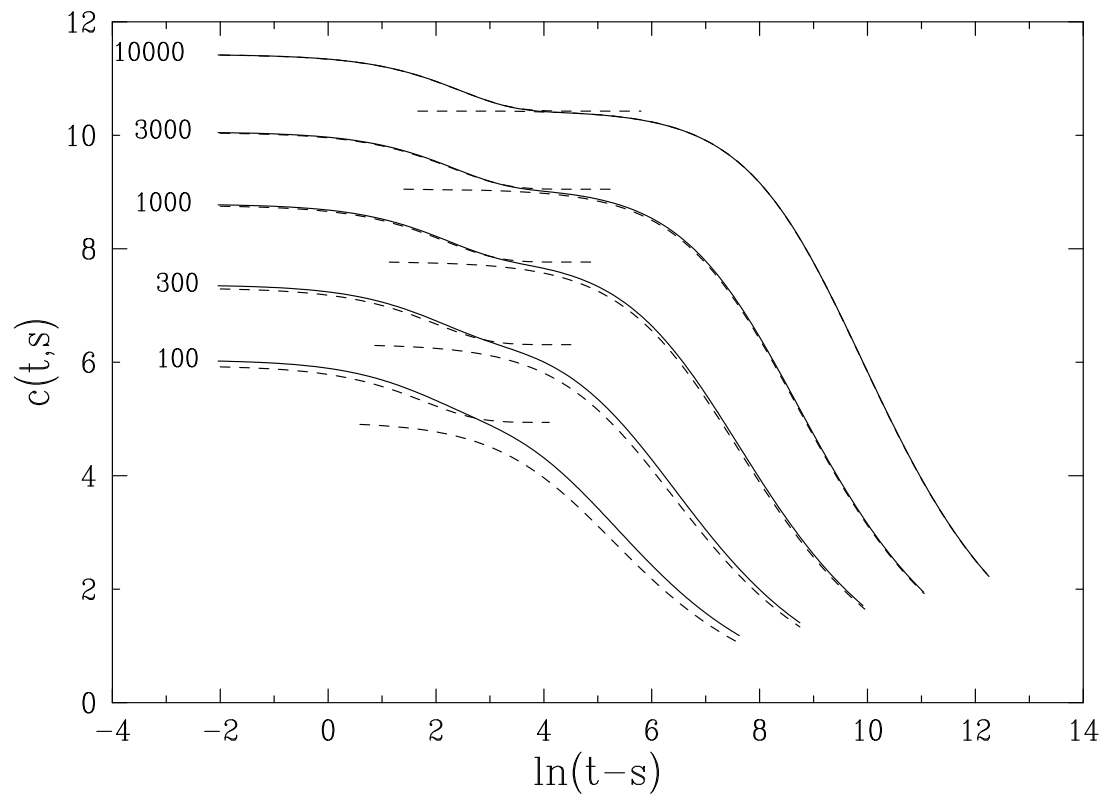

Figure 1: Plot of the zero-temperature correlation function $c(t, s)$ against $\ln (t-s)$, for different values of the waiting time $s$, indicated on the curves. Full lines: exact numerical results. Dashed lines: left ( $\beta$-regime): eq. (6.20); right ( $\alpha$-regime): scaling law (6.18), with plateau value $(6.19)$.

First, one finds

$$
\left(\frac{\partial c}{\partial s}\right)_{\mathrm{pl}}(s) \approx \frac{\Lambda(s)^{2} \mathrm{e}^{-\Lambda(s)}}{\Lambda(s)^{2} \mathrm{e}^{-\Lambda(s)} I(\Lambda(s))+1-\Lambda(s)}=\frac{\Lambda(s)-1}{t_{\mathrm{eq}}[\Lambda(s)]}
$$

where $t_{\text {eq }}[\Lambda(s)]$ is obtained from eq. (5.11) by replacing $\Lambda_{\text {eq }}$ by $\Lambda(s)$. Though the expression (6.21) is simple, since it can be obtained by replacing $\Lambda_{\mathrm{eq}}$ by $\Lambda(s)$ in the equilibrium result (5.19), its derivation is not.

The values of $r_{\mathrm{pl}}^{ \pm}(s)$, obtained along the same lines, have the more intricate expressions

$$
r_{\mathrm{pl}}^{+}(s) \approx \frac{\Lambda(s)^{2} \mathrm{e}^{-\Lambda(s)}-\Lambda(s) A\left(\Lambda(s), \Lambda_{\mathrm{eq}}\right)\left((\Lambda(s)-1) \mathrm{e}^{-\Lambda(s)} I(\Lambda(s))-1+\frac{1}{\Lambda(s)^{2}}\right)}{\Lambda(s)^{2} \mathrm{e}^{-\Lambda(s)} I(\Lambda(s))+1-\Lambda(s)},
$$




$$
r_{\mathrm{pl}}^{-}(s) \approx \frac{\Lambda(s)^{2} \mathrm{e}^{-\Lambda(s)}-\Lambda(s) A\left(\Lambda(s), \Lambda_{\mathrm{eq}}\right)\left(\Lambda(s) \mathrm{e}^{-\Lambda(s)} I(\Lambda(s))-1-\frac{1}{\Lambda(s)}+\frac{1}{\Lambda(s)^{2}}\right)}{\Lambda(s)^{2} \mathrm{e}^{-\Lambda(s)} I(\Lambda(s))+1-\Lambda(s)} .
$$

The scaling result (6.18) implies that the fluctuation-dissipation ratios $X^{ \pm}(t, s)$ stay constant and equal to their plateau values

$$
X_{\mathrm{pl}}^{ \pm}(s)=\frac{r_{\mathrm{pl}}^{ \pm}(s)}{(\partial c / \partial s)_{\mathrm{pl}}(s)}
$$

throughout the non-equilibrium $\alpha$-regime, i.e., for $t-s \gg 1$. Eqs. (6.21, 6.22) lead to

$$
\begin{aligned}
& X_{\mathrm{pl}}^{+}(s) \approx 1-\frac{\mathrm{e}^{\Lambda(s)} A\left(\Lambda(s), \Lambda_{\mathrm{eq}}\right)}{\Lambda(s)}\left((\Lambda(s)-1) \mathrm{e}^{-\Lambda(s)} I(\Lambda(s))-1+\frac{1}{\Lambda(s)^{2}}\right) \\
& X_{\mathrm{pl}}^{-}(s) \approx 1-\frac{\mathrm{e}^{\Lambda(s)} A\left(\Lambda(s), \Lambda_{\mathrm{eq}}\right)}{\Lambda(s)}\left(\Lambda(s) \mathrm{e}^{-\Lambda(s)} I(\Lambda(s))-1-\frac{1}{\Lambda(s)}+\frac{1}{\Lambda(s)^{2}}\right)
\end{aligned}
$$

These expressions interpolate between the equilibrium values $X_{\mathrm{pl}}^{ \pm}(s)=1$ for $s \gg t_{\mathrm{eq}}$, and the non-trivial values

$$
\begin{aligned}
& X_{\mathrm{pl}}^{+}(s) \approx \frac{1}{\Lambda(s)}+\left(1-\frac{1}{\Lambda(s)^{2}}\right) \frac{\mathrm{e}^{\Lambda(s)}}{\Lambda(s) I(\Lambda(s))} \approx 1-\frac{2}{\Lambda(s)^{2}}-\frac{2}{\Lambda(s)^{3}}-\frac{12}{\Lambda(s)^{4}}+\cdots, \\
& X_{\mathrm{pl}}^{-}(s) \approx\left(1+\frac{1}{\Lambda(s)}-\frac{1}{\Lambda(s)^{2}}\right) \frac{\mathrm{e}^{\Lambda(s)}}{\Lambda(s) I(\Lambda(s))} \approx 1-\frac{3}{\Lambda(s)^{2}}-\frac{3}{\Lambda(s)^{3}}-\frac{15}{\Lambda(s)^{4}}+\cdots
\end{aligned}
$$

at zero temperature, and more generally in the aging regime, i.e., for $s \ll t_{\text {eq }}$.

Figure 2 illustrates the above predictions. The zero-temperature plateau values $X_{\mathrm{pl}}^{ \pm}(s)$ are plotted against $\ln s$, for $s$ up to $10^{5}$. The full lines show the limit values $\lim _{t \rightarrow \infty} X^{ \pm}(t, s)$, obtained by extrapolating the numerical solutions of eqs. (2.4, 2.17, 2.30, 2.37). The dashed lines show the analytical predictions (6.25), whose accuracy becomes extremely high for the larger values of the waiting time $s$.

The above results can be compared with the expression for the zero-temperature plateau value of the fluctuation-dissipation ratio associated with energy fluctuations, derived in ref. [23]:

$$
X_{\mathrm{pl}}^{\mathrm{energy}}(s) \approx \frac{\Lambda(s)(\Lambda(s)-1)}{\Lambda(s)^{2} \mathrm{e}^{-\Lambda(s)} I(\Lambda(s))+(\Lambda(s)-1)^{2}} \approx 1-\frac{2}{\Lambda(s)^{2}}-\frac{4}{\Lambda(s)^{3}}-\frac{6}{\Lambda(s)^{4}}+\cdots
$$

The expressions (6.25, 6.26) of the three fluctuation-dissipation ratios are very similar. All these ratios asymptotically converge to the value 1 , characteristic of equilibrium, albeit 
with large correction terms, which only decay as $1 / \Lambda(s)^{2} \approx 1 /(\ln s)^{2}$, i.e., logarithmically slow with the waiting time $s$. More surprisingly, in the present case of density fluctuations, the difference

$$
X_{\mathrm{pl}}^{+}(s)-X_{\mathrm{pl}}^{-}(s) \approx \frac{1}{\Lambda(s)}\left(1-\frac{\mathrm{e}^{\Lambda(s)}}{\Lambda(s) I(\Lambda(s))}\right) \approx \frac{1}{\Lambda(s)^{2}}+\frac{1}{\Lambda(s)^{3}}+\frac{3}{\Lambda(s)^{4}}+\cdots,
$$

which is entirely due to the discontinuous slope of the Metropolis acceptance rate [see eq. (2.26)], and hence dependent on our choice of a microscopic dynamics for the model, exhibits the very same logarithmically slow fall-off in $1 /(\ln s)^{2}$.

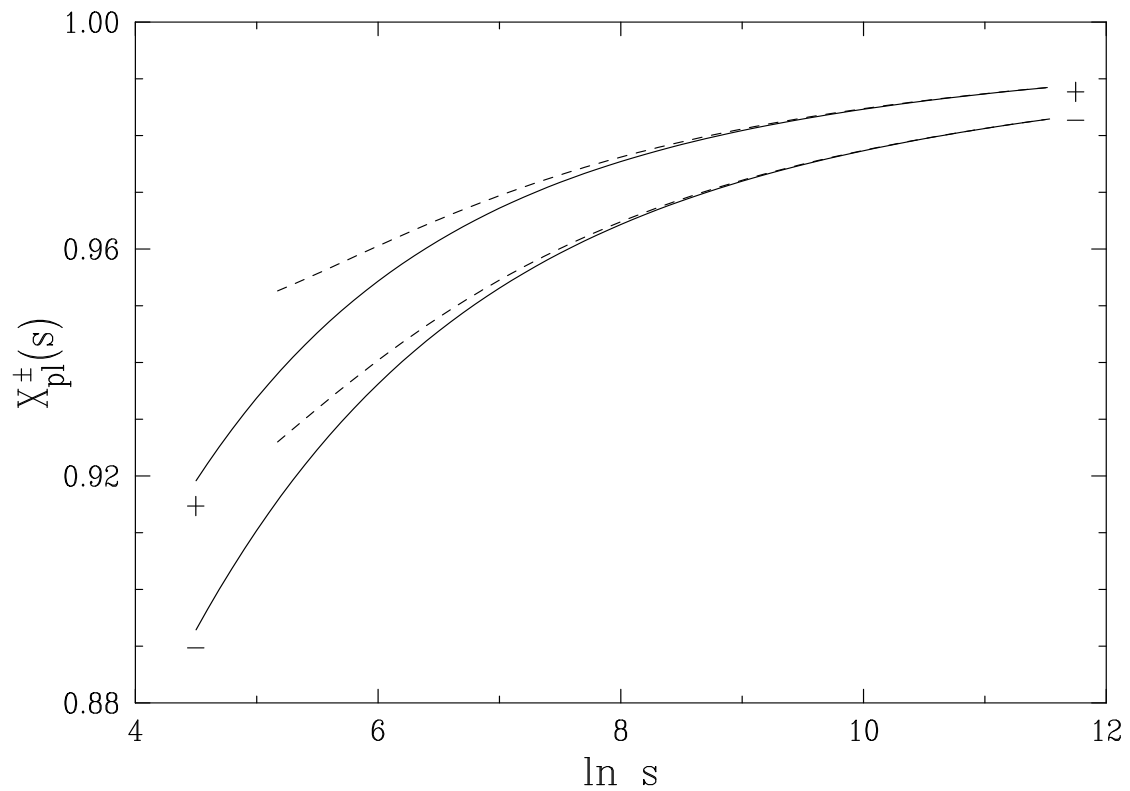

Figure 2: Plot of the zero-temperature plateau values $X_{\mathrm{pl}}^{+}(s)$ (upper data) and $X_{\mathrm{pl}}^{-}(s)$ (lower data), against $\ln s$. Full lines: exact numerical results. Dashed lines: analytical predictions (6.25).

To close up, we give a more detailed account of the dependence on both time scales $s$ and $t$ of the scaling law (6.18):

- At zero temperature, and more generally in the aging regime $\left(s \ll t_{\mathrm{eq}}\right)$, we have

$$
\alpha(\Lambda, \infty)=\frac{\Lambda^{2} \mathrm{e}^{-\Lambda} I(\Lambda)+1}{\Lambda\left(\Lambda^{2} \mathrm{e}^{-\Lambda} I(\Lambda)+1-\Lambda\right)} \approx \frac{1}{2}+\frac{1}{2 \Lambda}-\frac{1}{\Lambda^{2}}-\frac{7}{2 \Lambda^{3}}+\cdots,
$$

hence

$$
\begin{aligned}
\Phi(\Lambda(s), \infty) & \approx\left(\Lambda(s) \mathrm{e}^{\Lambda(s)}\right)^{1 / 2}\left(1+\frac{1}{\Lambda(s)}+\frac{9}{4 \Lambda(s)^{2}}+\cdots\right) \\
& \approx s^{1 / 2} \ln s\left(1+\frac{\ln \ln s}{\ln s}+\frac{4 \ln \ln s-9}{4(\ln s)^{2}}+\cdots\right)
\end{aligned}
$$


Therefore, in the aging regime $\left(s, t \ll t_{\text {eq }}\right)$, the right-hand-side of the scaling law (6.18) roughly obeys a square-root behavior, namely

$$
\frac{\Lambda(t)}{\Lambda(s)} \frac{\Phi(\Lambda(s), \infty)}{\Phi(\Lambda(t), \infty)} \approx\left(\frac{s}{t}\right)^{1 / 2}\left(1+\frac{1}{4}\left(\frac{1}{(\ln t)^{2}}-\frac{1}{(\ln s)^{2}}\right)+\cdots\right) .
$$

- In the opposite limit $\left(s \gg t_{\mathrm{eq}}\right)$, describing the convergence towards equilibrium, the scaling function $\Phi$ blows up exponentially, as

$$
\Phi\left(\Lambda(s), \Lambda_{\mathrm{eq}}\right) \approx C\left(\Lambda_{\mathrm{eq}}\right) \mathrm{e}^{s / t_{\mathrm{eq}}},
$$

with an $s$-independent prefactor. In this regime, the scaling law (6.18) therefore exhibits an exponential decay,

$$
\frac{\Lambda(t)}{\Lambda(s)} \frac{\Phi\left(\Lambda(s), \Lambda_{\mathrm{eq}}\right)}{\Phi\left(\Lambda(t), \Lambda_{\mathrm{eq}}\right)} \approx \mathrm{e}^{-(t-s) / t_{\mathrm{eq}}}
$$

characteristic of equilibrium properties.

\section{Summary and discussion}

The present work, a sequel of refs. [10, 21, 23], is devoted to a thorough study of the density correlation and response functions and of the associated fluctuation-dissipation ratios for the Backgammon model, a mean-field dynamical model introduced in the context of glassy dynamics [8]. We thus provide analytical predictions for the most salient findings of Franz and Ritort [22] for the relaxational dynamics of the density fluctuations of the model, both at equilibrium and away from equilibrium.

Let us first summarize our main results. Consider the situation at equilibrium and low temperature. The correlations in the Backgammon model possesses a spectrum of relaxation times with an exponential separation of time scales, between a $\beta$-regime of fast relaxation, involving the microscopic time scale, and a slow $\alpha$-regime, characterized by the relaxation time $t_{\mathrm{eq}} \approx 2 \mathrm{e}^{\Lambda_{\mathrm{eq}}} / \Lambda_{\mathrm{eq}} \approx 2 \mathrm{e}^{\beta} / \beta^{2}$, where $\Lambda_{\mathrm{eq}} \approx \beta$ is the fugacity of the system. The two-time correlation and response functions are stationary, i.e., they only depend on the time difference $\theta=t-s$. The density correlation function reads [see eq. (5.18)]

$$
c_{\mathrm{eq}}(\theta) \approx \underbrace{\mathrm{e}^{-\theta / \Lambda_{\mathrm{eq}}}}_{\beta \text {-regime }}+\underbrace{\left(\Lambda_{\mathrm{eq}}-1\right) \mathrm{e}^{-\theta / t_{\mathrm{eq}}}}_{\alpha \text {-regime }} .
$$

This expression accounts for the superposition of the fast $\beta$-relaxation $\left(\theta \sim 1\right.$ or $\left.\Lambda_{\mathrm{eq}}\right)$, and the slow $\alpha$-relaxation $\left(\theta \sim t_{\text {eq }}\right)$. As a consequence, eq. (7.1) exhibits a well-defined plateau value [see eq. (5.19)]

$$
\left(c_{\mathrm{eq}}\right)_{\mathrm{pl}} \approx \Lambda_{\mathrm{eq}}-1
$$


throughout the crossover between both regimes, i.e., for $1 \ll \theta \ll t_{\text {eq }}$. The same conclusions hold for the response, with

$$
r_{\mathrm{eq}}(\theta)=-\frac{\mathrm{d}}{\mathrm{d} \theta} c_{\mathrm{eq}}(\theta)
$$

which expresses the fluctuation-dissipation theorem.

One of the most striking outcomes of refs. [21, 23], further extended in the present work, is that the exponential separation of fast $(\beta)$ and slow $(\alpha)$ modes still holds in a generic non-equilibrium situation. This simplifying feature allows an analytical treatment of the non-equilibrium $\alpha$-regime at low temperature. Throughout this regime, we predict that the density correlation and response functions obey multiplicative scaling laws of the form [see eq. (6.18)]

$$
\frac{c(t, s)}{c_{\mathrm{pl}}(s)} \approx \frac{r^{ \pm}(t, s)}{r_{\mathrm{pl}}^{ \pm}(s)} \approx \frac{\partial c(t, s) / \partial s}{(\partial c / \partial s)_{\mathrm{pl}}(s)} \approx \frac{\Lambda(t)}{\Lambda(s)} \frac{\Phi\left(\Lambda(s), \Lambda_{\mathrm{eq}}\right)}{\Phi\left(\Lambda(t), \Lambda_{\mathrm{eq}}\right)}
$$

These scaling laws hold at low temperature $\left(t_{\text {eq }} \gg 1\right)$ and throughout the $\alpha$-regime $(s \gg 1, t-s \gg 1)$, irrespective of the ratios $s / t_{\mathrm{eq}}$ or $(t-s) / t_{\mathrm{eq}}$. When these ratios are small, i.e., at zero temperature, and more generally in the aging regime, the rightmost side of eq. (7.4) assumes an approximate inverse-square-root law $(s / t)^{1 / 2}$ [see eq. (6.30)]. Conversely, when the above ratios are large, i.e., in the regime of convergence towards equilibrium at a low but finite temperature, this rightmost side exhibits an exponential fall-off, of the form $\exp \left(-(t-s) / t_{\mathrm{eq}}\right)$, characteristic of the equilibrium $\alpha$-relaxation.

Moreover, in the case of the correlation function, the crossover between the nonequilibrium $\beta$ - and $\alpha$-regimes can be described by a simple additive formula, generalizing the equilibrium result (7.1), as

$$
c(t, s) \approx \underbrace{\mathrm{e}^{-(t-s) / \Lambda(s)}}_{\beta \text {-regime }}+\underbrace{(\Lambda(s)-1) \frac{\Lambda(t)}{\Lambda(s)} \frac{\Phi\left(\Lambda(s), \Lambda_{\mathrm{eq}}\right)}{\Phi\left(\Lambda(t), \Lambda_{\mathrm{eq}}\right)}}_{\alpha \text {-regime }} .
$$

This result, illustrated in Figure 1, amounts to saying that in the non-equilibrium $\beta$ regime the system is somehow at an instantaneous equilibrium, described by $\Lambda(s)$. The validity of such a description is related to the simplicity of the non-equilibrium plateau value [see eq. (6.19)]

$$
c_{\mathrm{pl}}(s)=\Lambda(s)-1 \text {. }
$$

The same holds for the derivative $\partial c(t, s) / \partial s$. On the contrary, for the response functions $r^{ \pm}(t, s)$, there is still a well-defined separation of a fast and a slow component away from equilibrium, but this separation is non-trivial, as it involves the plateau values $r_{\mathrm{pl}}^{ \pm}(s)$, which are complicated functions of $s$, even at zero temperature [see eqs. (6.22)]. 
It is worth comparing the results of this paper for the correlation and response of the density with those found for the correlation and response of the energy [21, 23]. First, as underlined in ref. 22], the density correlation has an appreciable variation over short time scales, i.e., in the $\beta$-regime, while the energy correlation remains essentially constant in this regime. Second, in the $\alpha$-regime, the scaling law (7.4) of the correlation and response functions of the density is very similar to that of the energy [23]. As it turns out, both scaling functions only differ by the prefactor $\Lambda(t) / \Lambda(s)$. Finally, in the present case of density fluctuations, because of the choice of a Metropolis dynamics, it is necessary to introduce two response functions, $r^{ \pm}(t, s)$, and accordingly two fluctuation-dissipation ratios, $X^{ \pm}(t, s)$. This subtlety was not needed in the case of energy fluctuations. A surprising outcome of the present study is that the difference $X_{\mathrm{pl}}^{+}(s)-X_{\mathrm{pl}}^{-}(s)$, which reflects the choice of the microscopic dynamics of the model, persists in the $\alpha$-regime, where it exhibits a logarithmically slow fall-off [see eq. (6.27)].

As can be seen on these results, the Backgammon model is helpful in giving clearcut analytical answers to some recurrent questions in the ongoing investigation of the slow dynamics of non-equilibrium systems. This knowledge in turn is of interest to see whether one can discriminate between non-equilibrium systems by their relaxational behavior, encoded in the correlation and response, or equivalently in the behavior of the fluctuation-dissipation ratio. Thus, in the present case, is the Backgammon model more akin to a coarsening system, or to a mean-field spin-glass model, or yet to another case?

In some respects, the quantity $\Lambda(t)$, which is omnipresent in our description of the dynamics of the model, plays the role of the mean domain size $L(t)$, which is the only characteristic length scale in a system undergoing phase ordering [24, 25]. Another common salient feature of the present model and of coarsening systems is that the scaling form (7.4) of the correlation function $c(t, s)$ involves the ratio of a function of $\Lambda(t)$ to the same function of $\Lambda(s)$. On the other hand, as far as the fluctuation-dissipation ratio is concerned, there is a maximal discrepancy between the present model at zero temperature, where both fluctuation-dissipation ratios $X^{ \pm}(t, s)$ go asymptotically to unity (with a large logarithmic correction, illustrated in Figure 2), and coarsening systems, for which the fluctuation-dissipation ratio is observed to go to zero [26, 27, 28]. (The interpretation of the latter behavior is that the long-time response comes from the movement of the domain walls, and therefore becomes more and more negligible as the domains grow [27, 28].) This discrepancy is actually due to the fact that the critical temperature of the Backgammon model is $T_{c}=0$. As a consequence, the low-temperature phase, where aging persists forever, is reduced to the critical point of the model. More generally, as will be presented elsewhere [29], the fluctuation-dissipation ratio exhibits special features for systems quenched to their critical point. 


\section{Acknowledgements}

We wish to thank S. Franz and F. Ritort for interesting discussions during the elaboration of this work. 


\section{A Derivation of non-equilibrium plateau values}

In this Appendix, we present the method used in subsection 6.2 for the evaluation of the plateau values $r_{\mathrm{pl}}^{ \pm}(s)$ and $(\partial c / \partial s)_{\mathrm{pl}}(s)$ in the generic non-equilibrium situation.

Let us take the example of the latter quantity. First, eq. (3.11) implies

$$
\left(\frac{\partial c}{\partial s}\right)_{\mathrm{pl}}(s)=-\Lambda(s)\left(Y_{\zeta}\right)_{\mathrm{pl}}(s)
$$

Second, $\left(Y_{\zeta}\right)_{\mathrm{pl}}(s)$ can be estimated by performing a Laplace transform on eqs. (3.3, 3.14). We thus obtain after some algebra

$$
\left(\frac{\partial c}{\partial s}\right)_{\mathrm{pl}}(s) \approx-\Lambda(s) \frac{\Lambda(s) S_{\zeta}(s)+(\Lambda(s)-1) f_{1}(s)}{\Lambda(s)^{2} \mathrm{e}^{-\Lambda(s)} I(\Lambda(s))+1-\Lambda(s)},
$$

with

$$
S_{\zeta}(s)=\Lambda(s) \int_{0}^{1} \mathrm{~d} x \frac{\mathrm{e}^{-\Lambda(s) x}}{1-x} Z(x, s, s),
$$

where $Z(x, s, s)$ is given in eq. (3.4) in terms of the generating series $F(x, s)$ of the occupation probabilities.

Generalizing the equilibrium results derived in Sec. 5, we anticipate that $S_{\zeta}(s)$ is exponentially small in $\Lambda(s)$. As a consequence, it is not legitimate to estimate $F(x, s)$ by just replacing $\Lambda_{\text {eq }}$ by $\Lambda(s)$ in the equilibrium result (5.4). In other words, we first have to derive a better evaluation of the occupation probabilities in the $\alpha$-regime at low temperature. This can be done by seeking a solution to the first partial differential equation of eq. (3.2) in the form

$$
F(x, s)=\frac{\Lambda(s)-1+\mathrm{e}^{(x-1) \Lambda(s)}}{\Lambda(s)}+\mathrm{e}^{(x-1) \Lambda(s)} \Delta(x, s),
$$

where the first term represents the occupation probabilities at the instantaneous equilibrium described by $\Lambda(s)$ [see eq. (5.4)], while the correction term $\Delta(x, s)$ is assumed to be small. Eq. (3.2) yields a differential equation for $\Delta(x, s)$, which can be integrated as

$$
\Delta(x, s)=-\frac{\mathrm{d} \Lambda(s)}{\mathrm{d} s} \int_{x}^{1} \mathrm{~d} y \frac{\mathrm{e}^{(1-y) \Lambda(s)}-(1-y) \Lambda(s)-1}{(1-y) \Lambda(s)} .
$$

This expression is proportional to $\mathrm{d} \Lambda(s) / \mathrm{d} s$, i.e., exponentially small in $\Lambda(s)$, as expected. The result (A.5) has several consequences.

- Setting $x=0$ in eq. (A.5), we obtain $\Delta(0, s) \approx-(\mathrm{d} \Lambda(s) / \mathrm{d} s) I(\Lambda(s)) / \Lambda(s)$. Remarkably enough, we thus recover the evolution equation (6.3) for $\Lambda(s)$ itself. 
- By expanding eq. (A.4) to second order in $(x-1)$, we obtain

$$
c(s, s)=\left\langle N_{1}(s)^{2}\right\rangle-1=\sum_{k \geq 0} k^{2} f_{k}(s)-1=\left.\frac{\partial^{2} F(x, s)}{\partial x^{2}}\right|_{x=1} \approx \Lambda(s)-\frac{\Lambda(s) A\left(\Lambda(s), \Lambda_{\mathrm{eq}}\right)}{2} .
$$

The second moment of the density fluctuations is thus given by the simple formula corresponding to the Poisson law of parameter $\Lambda(s)$, namely $c(s, s)=\left\langle N_{1}(s)^{2}\right\rangle-1=$ $\Lambda(s)$, based on the picture of an instantaneous equilibrium described by $\Lambda(s)$, up to an exponentially small correction, proportional to $\mathrm{d} \Lambda / \mathrm{d} s \approx A\left(\Lambda(s), \Lambda_{\mathrm{eq}}\right)$,

- Similarly, we can evaluate the initial values of the fluctuation-dissipation ratios $X^{ \pm}(s, s)$. We have indeed $r^{ \pm}(s, s) \approx \partial c(s, s) / \partial s \approx 1 / \Lambda(s)$, and

$$
\begin{aligned}
\frac{\partial c(s, s)}{\partial s}-r^{ \pm}(s, s) & =1-\mathrm{e}^{-\beta} f_{0}(s)+\frac{1}{\Lambda(s)}\left(f_{1}(s)-c(s, s)\right) \\
& \approx\left(\frac{1}{2}+\frac{1}{\Lambda(s)^{2}}\right) A\left(\Lambda(s), \Lambda_{\text {eq }}\right),
\end{aligned}
$$

hence

$$
X^{ \pm}(s, s) \approx 1-\left(\frac{1}{2}+\frac{1}{\Lambda(s)^{2}}\right) \Lambda(s) A\left(\Lambda(s), \Lambda_{\mathrm{eq}}\right) .
$$

The violation of the fluctuation-dissipation theorem at initial times is thus exponentially small, as it is again proportional to $\mathrm{d} \Lambda(s) / \mathrm{d} s$. We have in particular

$$
X^{ \pm}(s, s) \approx 1-\left(\frac{1}{2}+\frac{1}{\Lambda(s)^{2}}\right) \frac{1}{I(\Lambda(s))} \approx 1-\frac{\Lambda(s) \mathrm{e}^{-\Lambda(s)}}{2}
$$

at zero temperature.

- Finally, coming back to our main goal, i.e., the derivation of the plateau value of $(\partial c / \partial s)_{\mathrm{pl}}(s)$, we insert eqs. (A.4, A.5) into the expression (3.4) for $Z(x, s, s)$, and then perform the integral (A.3). All the integrals brought by the correction term $\Delta(x, s)$ can be expressed in terms of $I(\Lambda(s))$ and of elementary functions. We are thus left with

$$
\left(\frac{\partial c}{\partial s}\right)_{\mathrm{pl}}(s) \approx \frac{\Lambda(s)^{2} \mathrm{e}^{-\Lambda(s)}}{\Lambda(s)^{2} \mathrm{e}^{-\Lambda(s)} I(\Lambda(s))+1-\Lambda(s)},
$$

i.e., the result announced in eq. (6.21). The results (6.22) have been derived along the very same lines. 


\section{References}

[1] P. and T. Ehrenfest, The Conceptual Foundations of the Statistical Approach in Mechanics (Dover, 1990).

[2] P. and T. Ehrenfest, Phys. Zeit. 8 (1907), 311.

[3] M. Kac, in Probability and Related Topics in Physical Sciences, Lectures in Applied Mathematics, vol. 1 A (American Mathematical Society, 1959).

[4] F. Kohlrausch and E. Schrödinger, Phys. Zeit. 27 (1926), 306.

[5] M. Kac, Amer. Math. Monthly 54 (1947), 369.

[6] A.J.F. Siegert, Phys. Rev. 76 (1949), 1708.

[7] F.G. Hess, Amer. Math. Monthly 61 (1954), 323.

[8] F. Ritort, Phys. Rev. Lett. 75 (1995), 1190.

[9] For a recent review, see: J.P. Bouchaud, L.F. Cugliandolo, J. Kurchan, and M. Mézard, cond-mat/9702070, published in Spin Glasses and Random Fields, Directions in Condensed Matter Physics, vol. 12, edited by A.P. Young (World Scientific, 1998).

[10] C. Godrèche, J.P. Bouchaud, and M. Mézard, J. Phys. A 28 (1995), L 603.

[11] B.J. Kim, G.S. Jeon, and M.Y. Choi, Phys. Rev. Lett. 76 (1996), 4648.

[12] P. Bialas, Z. Burda, and D. Johnston, Nucl. Phys. B 493 (1997), 505.

[13] J.M. Drouffe, C. Godrèche, and F. Camia, J. Phys. A 31 (1998), L 19.

[14] A. Prados, J.J. Brey, and B. Sanchez-Rey, Phys. Rev. B 55 (1997), 6343.

[15] A. Lipowski, J. Phys. A 30 (1997), L 91.

[16] K.P.N. Murthy and K.W. Kehr, J. Phys. A 30 (1997), 6671.

[17] S.J. Cornell, K. Kaski, and R.B. Stinchcombe, Phys. Rev. B 44 (1991), 12263.

[18] L.F. Cugliandolo and J. Kurchan, J. Phys. A 27 (1994), 5749.

[19] S. Franz and F. Ritort, Europhys. Lett. 31 (1995), 507.

[20] S. Franz and F. Ritort, J. Stat. Phys. 85 (1996), 131. 
[21] C. Godrèche and J.M. Luck, J. Phys. A 29 (1996), 1915.

[22] S. Franz and F. Ritort, J. Phys. A 30 (1997), L 359.

[23] C. Godrèche and J.M. Luck, J. Phys. A 30 (1997), 6245.

[24] J.S. Langer, in Solids far from equilibrium, edited by C. Godrèche (Cambridge University Press, 1991).

[25] A.J. Bray, Adv. Phys. 43, 357 (1994).

[26] L.F. Cugliandolo, J. Kurchan, and L. Peliti, Phys. Rev. E 55 (1997), 3898.

[27] A. Barrat, Phys. Rev. E 57 (1998), 3629.

[28] L. Berthier, J.L. Barrat, and J. Kurchan, preprint cond-mat/9903091.

[29] C. Godrèche and J.M. Luck, in preparation. 